\title{
Polimorfismos de la región promotora del gen de la IL-10 y artritis reumatoide en una población colombiana
}

\author{
Olga María Moreno ${ }^{1}$, Clara Isabel González ${ }^{1}$, Diego Luis Saaibi ${ }^{2}$, William Otero ${ }^{3}$, \\ Reynaldo Badillo ${ }^{4}$, Javier Martín ${ }^{5}$, Gerardo Ramírez 1,2 \\ ${ }^{1}$ Laboratorio de Inmunología y Biología Molecular, Grupo de Inmunología y Epidemiología Molecular GIEM, \\ Facultad de Salud, Universidad Industrial de Santander, Bucaramanga, Colombia. \\ 2 Medicina Interna, Clínica y Centro Médico Carlos Ardila Lulle, Floridablanca, Colombia. \\ ${ }^{3}$ Hospital Universitario de Santander, Bucaramanga, Colombia. \\ ${ }^{4}$ Departamento de Medicina Interna, Facultad de Salud, Universidad Industrial de Santander, Bucaramanga, \\ ${ }^{5}$ Instituto de Parasitología y Biomedicina López-Neyra, IPB, Granada, España.
} Colombia.

Introducción. La artritis reumatoide es una enfermedad inflamatoria con un predominio de la actividad de las células TH1 CD4+. La interleucina-10, presente en altas concentraciones en suero y líquido sinovial de pacientes con artritis reumatoide, tiene una marcada actividad antinflamatoria, al mismo tiempo que estimula las células B y la generación de autoanticuerpos. La producción de la interleucina-10 está bajo control genético.

Objetivo. En este estudio analizamos los polimorfismos de la región promotora de la interleucina-10 -1082, -819 y -592 en pacientes con artritis reumatoide y en una población control, y su asociación con edad de inicio de la enfermedad, presencia y títulos de factor reumatoideo e historia de reemplazo articular.

Materiales y métodos. Se estudiaron 102 pacientes con artritis reumatoide y 102 controles. La genotipificación se realizó por reacción en cadena de la polimerasa, iniciador específico de secuencia. Los tres polimorfismos están en marcado desequilibrio de unión y forman tres haplotipos -1082A/-819C/-592C, -1082A/-819T/-592A y -1082G/-819C/-592C.

Resultados. No se encontró asociación de la artritis reumatoide con las diferentes variaciones alélicas, haplotípicas ni genotípicas del promotor de la interleucina-10. Tampoco se encontraron diferencias significativas con inicio de la enfermedad, presencia y títulos de factor reumatoideo e historia de reemplazo articular.

Conclusiones. La interleucina-10 es uno de los principales reguladores de la respuesta inmune y por lo tanto podría jugar un papel importante en la patogénesis de la artritis reumatoide; sin embargo, nuestros resultados no dan evidencia de una asociación genética entre los polimorfismos estudiados y el desarrollo o gravedad de la artritis reumatoide.

Palabras clave: interleucinas, interleucina-10, polimorfismo genético, artritis reumatoide, polimorfismo de nucleótido simple.

Polymorphisms of IL-10 gene promoter and rheumatoid arthritis in a Colombian population

Introduction. Rheumatoid arthritis is an inflammatory disease driven by $\mathrm{TH} 1 \mathrm{CD}^{+}$cells. Interleukin-10 is present in higher concentrations in serum and synovial fluid from patients with rheumatoid arthritis and has a marked anti-inflammatory activity. Furthermore, it is capable of stimulating B cells and increasing autoantibody production. Interleukin-10 synthesis is under genetic control.

Objective. Three polymorphisms of the promoter region were analyzed for interleukin-10 genes $-1082,-819$ and -592 . Subjects were patients with rheumatoid arthritis compared with a control population for these genes.

Material and methods. One hundred two patients with rheumatoid arthritis and 102 matched healthy controls were studied. The following data were taken from the rheumatoid arthritis patients: age of disease onset, presence and titers of rheumatoid factor, and history of replacement 
joint surgery. Genotypes were obtained by polymerase chain reaction and sequence-specific primer method. The three polymorphisms are in strong linkage-disequilibrium and form three haplotypes $-1082 \mathrm{~A} /-819 \mathrm{C} /-592 \mathrm{C},-1082 \mathrm{~A} /-819 \mathrm{~T} /-592 \mathrm{~A}$ y $-1082 \mathrm{G} /-819 \mathrm{C} /-592 \mathrm{C}$.

Results. No association was detected between Interleukin-10 alleles, haplotypes/genotypes and rheumatoid arthritis. No significant differences occurred between interleukin-10 polymorphisms and age of disease onset, presence and titer of rheumatoid factor and history of major joint replacement.

Conclusions. Interleukin-10 is an important regulator of the immune response and likely plays a role in the pathogenesis of rheumatoid arthritis. The current results suggested that Interleukin10 promoter polymorphisms were not important for development or severity of rheumatoid arthritis.

Key words: Interleukins; interleukin-10; polymorphism, genetic; arthritis, rheumatoid; polymorphism, single nucleotide.

La artritis reumatoide (AR) es una poliartritis inflamatoria sistémica que afecta a cerca del $1 \%$ de la población general con altos costos personales, sociales y económicos. Los genes de la cadena $\beta 1$ de los antígenos del complejo mayor de histocompatibilidad de clase II, especialmente el HLA-DR4, son los que más se han asociado con la susceptibilidad y gravedad de la enfermedad $(1,2)$. Sin embargo, se considera que la contribución de los genes localizados en el complejo HLA sólo explican entre 30 y $50 \%$ del componente genético en la AR (3). Las citocinas han sido asociadas con la patogénesis de la $A R$ y juegan un papel regulador y efector en la respuesta inflamatoria en esta patología $(4,5)$; por lo tanto, los polimorfismos en sus genes podrían influir el desarrollo o la gravedad de la enfermedad.

Se considera que en la AR el proceso inflamatorio es orquestado por las células TH1 CD4+ que estimulan los monocitos, macrófagos, fibroblastos sinoviales y otras células a producir citocinas como el factor de necrosis tumoral $\alpha$ (TNF- $\alpha$ ), IL-1, IL-6, IL-15, IL-17 y metaloproteinasas que producen el daño tisular. En respuesta a esta reacción proinflamatoria se activan mecanismos antinflamatorios producidos por las células $\mathrm{TH} 2 \mathrm{CD}^{+}$en un intento por controlar la autorreactividad, los

\footnotetext{
Correspondencia:

Gerardo Ramírez, Laboratorio de Inmunología y Biología Molecular, Facultad de Salud, Universidad Industrial de Santander, Bucaramanga, Santander, Colombia.

Cra 32 No. 29-31, oficina 407. Teléfono: 6393163 , fax: 6393475.

gemensu@yahoo.com
}

Recibido: 16/08/06; aceptado: 05/12/06 cuales incluyen la producción de IL-10, IL-13, IL-4, factor de transformación de crecimiento (TGF- $\beta$ ), así como antagonistas de la reacción inflamatoria tales como los receptores solubles de TNF- $\alpha$ y el antagonista del receptor de la IL1 (IL-1Ra) $(4,6,7)$.

Se ha demostrado que la IL-10 suprime la inflamación en diversos modelos de enfermedad inflamatoria, incluida la artritis inducida por colágeno, la uveítis, la queratitis, la pancreatitis y la injuria pulmonar, inhibiendo la producción de mediadores proinflamatorios y la generación de moléculas coestimuladoras $(8,9)$. En pacientes con AR, la IL-10 se encuentra en altas concentraciones en suero y líquido sinovial $(10,11)$. Además de sus acciones inmunosupresoras, la IL10 estimula las células B aumentando la expresión de moléculas del complejo mayor de histocompatibiidad clase II, la producción de inmunoglobulinas y la replicación del ADN (12).

El gen de la IL-10 se localiza en el cromosoma 1(q31-q32) y es altamente polimórfico (13). Se sabe que los polimorfismos en la región reguladora de los genes de las citocinas pueden influir en su expresión. En la región promotora se han descrito al menos 11 polimorfismos de nucleótido simple (SNP); los ubicados en las posiciones -1082 (G/ A), $-819(\mathrm{C} / \mathrm{T})$ y $-592(\mathrm{C} / \mathrm{A})$ son los más estudiados; éstos se encuentran en fuerte desequilibrio de unión y generan sólo tres haplotipos: GCC ( $G$ en la posición -1082, C en la posición -819 y C en la posición -592), ACC y ATA. También se han estudiado con frecuencia en estudios de asociación un polimorfismo de nucleótido simple en la 
posición -2849 y dos microsatélites IL-10G e IL10R situados a $1,1 \mathrm{~kb}$ y $4 \mathrm{~kb}$ corriente arriba del sitio de iniciación de la transcripción $(14,15)$. Mediante pruebas in vitro se ha demostrado que la presencia del alelo $G$ en -1082 y en -2849 y de algunos alelos del tipo de repeticiones en tándem cortas (STR del inglés short tandem repeats), se han asociado con aumento en la transcripción de la proteína $(16,17)$. Se ha postulado que la sinovitis crónica observada en la AR refleja un desequilibrio en la producción de citocinas proinflamatorias y antinflamatorias, y que los altos niveles de IL-10 no son suficientes para contrarrestar el exceso de moléculas inflamatorias $(18,19)$. Basados en estas observaciones decidimos adelantar un estudio de asociación de los polimorfismos -1082, -819 y -592 en una población étnicamente diferente a las caucásica y asiática para contribuir a la comprensión del papel de la IL-10 en la artritis reumatoide.

\section{Materiales y métodos}

\section{Pacientes y controles}

La muestra incluyó 102 individuos con artritis reumatoide que cumplían con los criterios de clasificación del Colegio Americano de Reumatología (20) y 102 controles sanos procedentes del mismo entorno de los pacientes, similares en edad y sexo, sin enfermedad reumática aparente ni historia familiar de artritis reumatoide. El estudio fue aprobado por el Comité de Ética de la Universidad Industrial de Santander $y$ todos los participantes firmaron un consentimiento informado. Se incluyeron sólo individuos adultos mayores de 16 años que no hubieran recibido transfusiones sanguíneas en los seis meses anteriores y se estipuló que los controles no fueran familiares entre sí ni de los casos.

\section{Información clínica de los pacientes}

Todos los pacientes respondieron un cuestionario en el momento de su reclutamiento, en el cual se registraron datos demográficos como la edad, el sexo, la edad de presentación de la AR, la duración de la enfermedad y los antecedentes de cirugía de reemplazo articular mayor, además de los criterios de clasificación del Colegio Americano de Reumatología.

\section{Aislamiento del ADN}

El ADN genómico se aisló de sangre periférica anticoagulada usando el método de salting out descrito por Miller et al. (21).

Tipificación de los polimorfismos de IL-10 -1802, -819 y-592. La genotipificación se realizó con el método de reacción en cadena de la polimerasa, iniciador específico de secuencia (PCR-SSP), descrito por Mullighan et al. (22). Este método se fundamenta en la utilización de iniciadores sentido $(F)$ y antisentido (R) que especifican el reconocimiento alélico durante la amplificación (bases subrayadas en el cuadro 1). Como controles internos de cada reacción se usaron iniciadores para amplificar un fragmento de $796 \mathrm{pb}$ no polimórfico del gen HLA DR $\beta 1$, usado para amplificaciones específicas de alelos o haplotipos de tamaños menores de $600 \mathrm{pb}$ (22). Los tres polimorfismos determinados están muy cerca uno de otro y forman uniformemente tres haplotipos: GCC, ACC y ATA; esto permite determinarlos como tales mediante la combinación de los iniciadores descritos en el cuadro 1. La reacción en cadena de la polimerasa para cada haplotipo se hizo por separado y la presencia de cada haplotipo se confirmó en electroforesis en gel de agarosa $(1,5 \%)$, coloración con bromuro de etidio y revelado con luz ultra violeta por visualización simultánea de una banda correspondiente al control y una banda correspondiente al haplotipo amplificado. La combinación de los diferentes iniciadores permitió, además, controlar la especificidad de la amplificación. Los detalles técnicos para la detección de cada haplotipo están en el cuadro 1.

\section{Determinación de factor reumatoideo}

El factor reumatoideo fue determinado en el suero con el método de aglutinación en látex (HUMANTEX RF, Germany). Los títulos de $\geq 20$ $\mathrm{UI} / \mathrm{ml}$ se consideraron como positivos. La prueba se realizó bajo las mismas condiciones en todo el grupo de estudio, incluidos los controles.

\section{Gravedad de la enfermedad}

Se tomó como criterio de gravedad de la enfermedad la presencia de cirugía de reemplazo articular mayor, usualmente en la rodilla o la cadera. 
Cuadro 1. Secuencias iniciadoras, condiciones de amplificación e identificación de los polimorfismos con PCR-SSP.

\begin{tabular}{|c|c|c|c|}
\hline Haplotipo & Iniciadores & $\begin{array}{l}\text { Condiciones de } \\
\text { amplificación }\end{array}$ & $\begin{array}{l}\text { Tamaño } \\
\text { de fragmentos } \\
\text { amplificados }\end{array}$ \\
\hline$\underline{A C C}$ & $\begin{array}{l}\text { F 5' -CTA CTA AGG CTT CTT TGG GAA- 3' } \\
\text { R 5' -CCA GAG ACT GGC TTC CTA CAG } \underline{\text { G- }} \text { ', }\end{array}$ & $\begin{array}{l}96^{\circ} \mathrm{C} 25 \mathrm{~s}, 70^{\circ} \mathrm{C} 5 \mathrm{~s}, 72^{\circ} \mathrm{C} \\
45 \mathrm{~s}: 5 \text { ciclos. }\end{array}$ & $529 \mathrm{pb}$ \\
\hline$\underline{\text { ATA }}$ & $\begin{array}{l}\text { F } 5 \text { '-CTA CTA AGG CTT CTT TGG GAG- 3' } \\
\text { R 5' -CAA ACT GAG GCA CAG AGA TG- 3' }\end{array}$ & $\begin{array}{l}96^{\circ} \mathrm{C} 25 \mathrm{~s}, 65^{\circ} \mathrm{C} 50 \mathrm{~s} \\
72^{\circ} \mathrm{C} 45 \mathrm{~s}: 21 \text { ciclos. }\end{array}$ & $303 p b$ \\
\hline$\underline{\mathrm{GCC}}$ & $\begin{array}{l}\text { F 5' -CTA CTA AGG CTT CTT TGG GAG- 3' } \\
\text { R 5' -CAA ACT GAG GCA CAG AGA T互- 3' }\end{array}$ & $\begin{array}{l}96^{\circ} \mathrm{C} 25 \mathrm{~s}, 55^{\circ} \mathrm{C} 60 \mathrm{~s} \\
72^{\circ} \mathrm{C} 120 \mathrm{~s}: 4 \text { ciclos. }\end{array}$ & $304 \mathrm{pb}$ \\
\hline DR $\beta 1$ & $\begin{array}{l}\text { F 5' -TGC CAA GTG GAG CAC CCA A- 3' } \\
\text { R 5' -GCA TCT TGC TCT GTG CAG AT- 3' }\end{array}$ & & $796 \mathrm{pb}$ \\
\hline
\end{tabular}

\section{Análisis estadístico}

Las frecuencias alélicas, haplotípicas y genotípicas se establecieron por conteo directo. La comparación de las frecuencias de haplotipos y de genotipos entre los grupos se realizó por medio de la prueba de ji cuadrado y mediante el uso de tablas de contingencia y la prueba de Fisher (para valores de frecuencias menores de 5) (23). Se estableció un intervalo de confianza del $95 \%$ $(p<0,05)$. La determinación de la asociación de los polimorfismos con otros parámetros de la enfermedad como edad de inicio (inicio temprano $<45$ años, inicio tardío $>45$ años), factor reumatoideo, títulos de factor reumatoideo (títulos bajos: $20 \mathrm{Ul} / \mathrm{ml}-<192 \mathrm{Ul} / \mathrm{ml}$, títulos altos $\geq 192$ $\mathrm{UI} / \mathrm{ml}$ ) y la necesidad de cirugía de reemplazo articular, se llevó a cabo mediante la prueba de ji cuadrado y el uso de tablas $2 \times 2$; la prueba exacta de Fisher se aplicó cuando los valores en la tabla fueron menores de 5 (23). La razón de disparidad (OR) y los intervalos de confianza fueron establecidos en un intervalo del $95 \%(p<0,05)$ en EPIINFO 6. Los análisis de equilibrio de HardyWeinberg (EHW) y del coeficiente de fijación de Wright Fst para determinar el grado de estructuración de la población se realizaron mediante el paquete estadístico Arlequín, versión 2000 (24). El resultado del coeficiente de Wright se interpreta así: 0 a 0,05, poca estructura; 0,05 a 0,15 , estructura moderada; 0,15 a 0,25, mucha estructura, y 0,25 a 1, gran estructura (25).

\section{Resultados}

Las características clínicas de los pacientes con AR se resumen en el cuadro 2. El promedio de edad del grupo control $(50,33 \pm 12,57$ años $)$ no mostró diferencias significativas con el de los pacientes (51,05 $\pm 12,87$ años). La edad promedio de inicio de la AR en el grupo estudiado fue de $39,2 \pm 13,85$ años. Las frecuencias genotípicas de los polimorfismos -819 y -592 se encontraron en equilibrio de Hardy-Weinberg en ambos grupos; sin embargo, las de -1082 en el grupo control mostraron desviación en relación con las esperadas $(p=0,026)$ por presencia de un exceso de heterocigotos $A G$ a expensas de una reducción de los homocigotos GG (55/45 y 6/11, obs/esp, respectivamente); en el grupo de casos se encontraron en equilibrio. El coeficiente de variación Fst reveló estructuración moderada de la población ( $F s t=0,06047)(25)$.

$\mathrm{Al}$ igual que en otros estudios, se encontró ligamiento completo de los polimorfismos $-819 \mathrm{C} /$ $-592 \mathrm{C}$ y-819T/-592A, por lo que sólo se hallaron los haplotipos ACC, ATA y GCC (15). La distribución de las frecuencias alélicas, haplotípicas, genotípicas y de los polimorfismos analizados en pacientes con AR y en el grupo 
control se muestran en el cuadro 3 . No se encontraron diferencias significativas en las frecuencias entre los grupos. En el cuadro 4 se muestra la frecuencia de los alelos, haplotipos y genotipos de IL-10 en pacientes con AR en relación con el inicio de la enfermedad (temprana o tardía), la presencia de factor reumatoideo, títulos altos o bajos de factor reumatoideo y los antecedentes

Cuadro 2. Información demográfica y clínica de los pacientes con artritis reumatoidea y el grupo control.

\begin{tabular}{lcc}
\hline Parámetro & Pacientes & Controles \\
\hline Número total de individuos & 102 & 102 \\
Proporción de sexos F/M (\%) & $86 / 16(84,3 / 15,7)$ & $86 / 16(84,3 / 15,7)$ \\
Edad (promedio años \pm DS) & $51,0 \pm 12,8$ & $50,3 \pm 12,5$ \\
Edad de inicio (promedio años \pm DS)) & $39,2 \pm 13,8$ & \\
Duración de la enfermedad (Promedio años $\pm D S)$ & $12,2 \pm 9,9$ & $97(95,0)$ \\
Historia de cirugía $n(\%)$ & $26(25,5)$ & $5(4,9)$ \\
FR $<$ de 20 Ul/ml (FR-) n (\%) & $36(35,3)$ & $5(100)$ \\
FR $\geq$ de 20 Ul/ml (FR+) $n(\%)$ & $66(64,7)$ & 0 \\
$\geq 20 ~ U l<192$ Ul/ml n (\%) & $30(45,5)$ & $27,20(20-48)$ \\
$\geq 192$ Ul/ml $n(\%)$ & $36(54,5)$ & \\
Promedio FR (Rango) Ul/ml & $304,39(20-3072)$ & \\
\hline
\end{tabular}

F/M: femenino/masculino, UI: Unidades Internacionales, DS: desviación standard, FR: factor reumatoideo.

Cuadro 3. Frecuencias alélicas, haplotípicas y genotípicas de los polimorfismos en el gen IL10, en pacientes con AR y el grupo control.

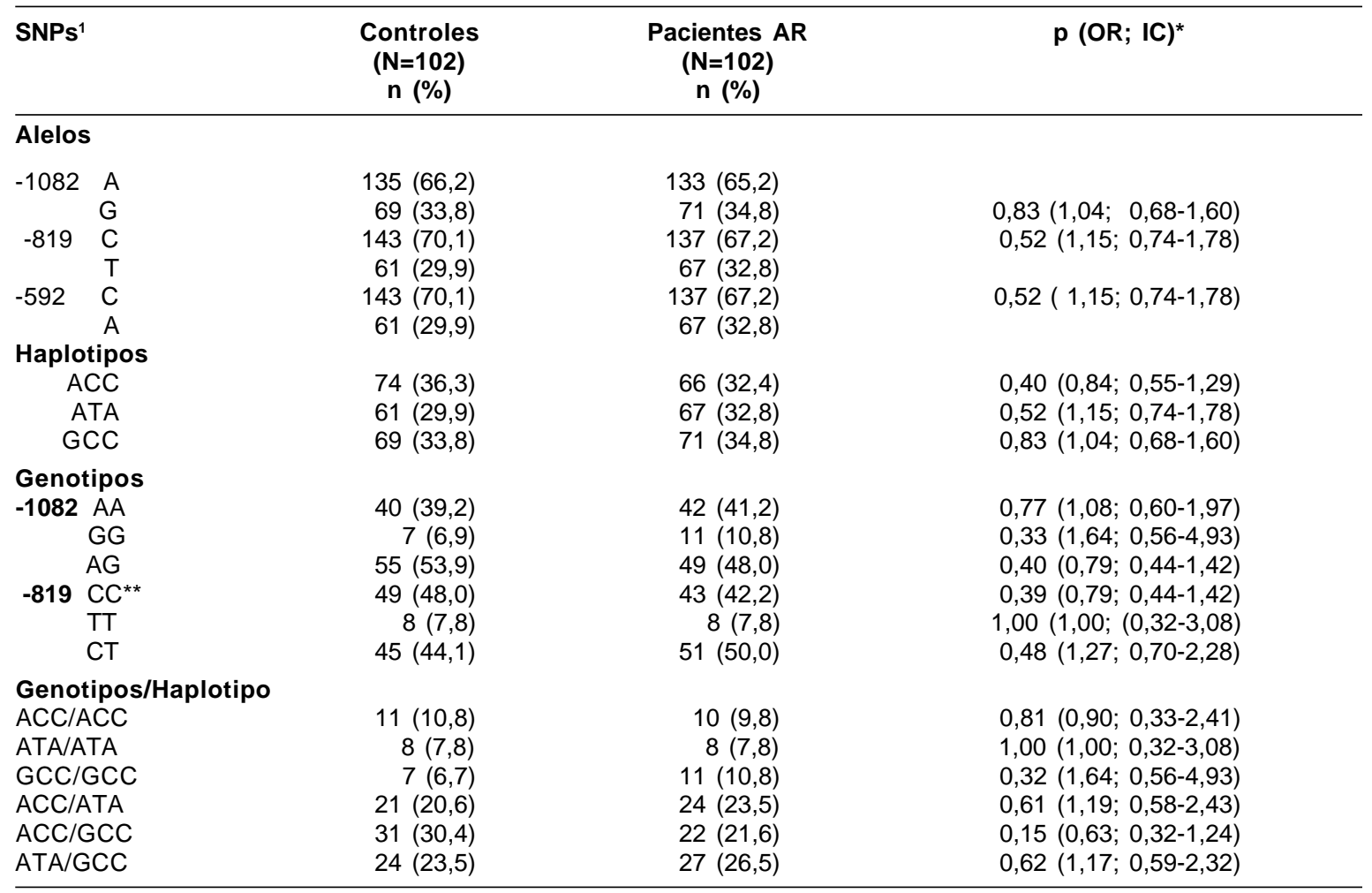

${ }^{*}$ Razón de disparidad (OR) e intervalo de confianza ( IC: 95\%). ${ }^{* *}$ Las frecuencias genotípicas de IL-10-590 son iguales a las de -819 por ligamiento completo de $-819 \mathrm{C}$ con $-590 \mathrm{C}$ y $-819 \mathrm{~T}$ con $-590 \mathrm{~A}$. Las comparaciones entre los grupos y los cálculos de las $p$ y de los OR se hicieron así: IL-10 -1082 G vs A, IL-10-819 y -592 C vs T y C vs A; los haplotipos y los genotipos se compararon cada uno vs todos los demás en cada caso.

${ }^{1}$ SNPs: polimorfismo de nucleótido único (Single Nucleotide Polymorphisms). 
Cuadro 4. Frecuencias alélicas, haplotípicas y genotípicas de los polimorfismos evaluados en el gen de la IL10 y su relación con edad de inicio, factor reumatoideo (FR) y necesidad de cirugía articular en los pacientes con AR.

\begin{tabular}{|c|c|c|c|c|c|c|c|c|}
\hline \multirow[t]{2}{*}{ SNP } & $\begin{array}{l}\text { Edad de inicio } \\
<45 \text { años } \\
(\mathrm{N}=69)\end{array}$ & $\begin{array}{l}\text { Edad de inicio } \\
\begin{array}{c}\geq 45 \text { años } \\
(\mathrm{N}=33)\end{array}\end{array}$ & $\begin{array}{l}\text { FR (-) negativo } \\
\qquad(\mathrm{N}=36)\end{array}$ & $\begin{array}{c}\text { FR (+) positivo } \\
(\mathrm{N}=66)\end{array}$ & $\begin{array}{c}\text { FR }(+)^{1} \\
\text { Títulos bajos } \\
(\mathrm{N}=30)\end{array}$ & $\begin{array}{c}\text { FR(+) })^{2} \\
\text { Títulos altos } \\
(\mathrm{N}=36)\end{array}$ & $\begin{array}{c}\text { Cirugía } \\
(-) \\
(\mathrm{N}=76)\end{array}$ & $\begin{array}{c}\text { Cirugía } \\
(+) \\
(\mathrm{N}=26)\end{array}$ \\
\hline & \multicolumn{8}{|c|}{ n (\%) } \\
\hline
\end{tabular}

\section{Alelos $^{\text {a* }}$}

$-1082 \mathrm{~A}$

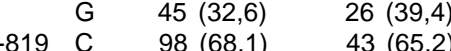

$\begin{array}{rrrr}\mathrm{T} & 44(31,9) & 23(34,8) \\ -592 \mathrm{C} & 98(68,1) & 43(65,2)\end{array}$

$-592 \mathrm{C} \quad 98(68,1) \quad 43(65,2)$

$44(31,9) \quad 23(34,8)$

Haplotipos*

ACC $49(35,5)$

ATA $\quad 44(31,9)$

GCC $\quad 45(32,6)$

Genotipos*

ACC/ACC $8(11,6)$

ATA/ATA $6(8,7)$

GCC/GCC $7(10,0)$

ACC/ATA $17(24,6)$

ACC/GCC $16(23,2)$

ATA/GCC $15(21,7)$

$$
\begin{array}{ll}
44 & (61,1) \\
28 & (38,9) \\
48 & (66,7) \\
24 & (33,3) \\
48 & (66,7) \\
24 & (33,3)
\end{array}
$$

$17(23,8)$

$23(34,8)$

$26(39,4)$

$2(6,1)$

$2(6,1)$

$4(12,1)$

$7(21,2)$

$6(18,2)$

$12(36,4)$

$\begin{array}{ll}89(67,4) & 39(65,0) \\ 43(32,6) & 21(35,0) \\ 89(67,4) & 43(71,7) \\ 43(32,6) & 17(28,3) \\ 89(67,4) & 43(71,7) \\ 43(32,6) & 17(28,3)\end{array}$

$50(69,4) \quad 100(65,8) \quad 35(67,3)$

$22(30,6) \quad 52(34,2) \quad 17(32,7)$

$46(63,9) \quad 101(66,4) \quad 35(67,3)$

$26(36,1) \quad 51(33,6) \quad 17(32,7)$

$46(63,9) \quad 101(66,4) \quad 35(67,3)$

$26(36,1) \quad 51(33,6) \quad 17(32,7)$

SNP: polimorfismo de nucleótido único (Single Nucleotide Polymorphisms)

${ }^{1} F R(+)$ Títulos bajos: $\geq 20 \mathrm{IU} / \mathrm{ml}$ a $<192 \mathrm{IU} / \mathrm{ml}$

${ }^{2}$ FR (+) Títulos altos: $\geq 192 \mathrm{IU} / \mathrm{ml}$

a Las frecuencias genotípicas individuales no se muestran, pero se analizaron para asociación en todos los parámetros.

*Las frecuencias entre los grupos y los análisis de asociación no fueron estadísticamente significativos.

de reemplazo articular. Si bien no se encuentraron diferencias significativas, se observó una mayor frecuencia del genotipo ACC/ACC con inicio temprano de la enfermedad $(11,5 \%$ vs. $6,1 \%)$ y presencia de factor reumatoideo (12,1\% vs. 5,6\%).

\section{Discusión}

La IL-10 es una citocina con potentes efectos sobre diversas poblaciones celulares y una acción reguladora sobre el sistema inmune $(8,17)$. Los niveles elevados de esta citocina parecen ser importantes en la patogenia del lupus eritematoso sistémico $(26,27)$, la enfermedad de Sjögren (28), el linfoma (29) y la sepsis (30), mientras que los niveles bajos de la IL-10 están relacionados con la enfermedad inflamatoria intestinal (31), la psoriasis (32) y el asma grave (33).

La información obtenida recientemente también sugiere que la IL-10 puede ser importante en la patogénesis de la AR. Los niveles de IL-10 aumentan tanto en suero como en líquido sinovial en pacientes con artritis reumatoide comparados con los controles (11). Los monocitos de sangre periférica de pacientes con artritis reumatoide, así como las células mononucleares del líquido sinovial producen espontáneamente más IL-10 que en individuos normales $(11,34)$. Los cultivos de células de membrana sinovial liberan espontáneamente IL-10 al igual que IL-1 y TNF $\alpha$ y la neutralización de la IL-10 da como resultado un aumento de la IL-1 y del TNF $\alpha$, poniendo en evidencia que la IL-10 actúa constitutivamente como supresor natural de estas citocinas en la AR (35). Por el contrario, la IL-10 tiene acciones proinflamatorias y estimula la producción de autoanticuerpos por parte de los linfocitos (8). En el suero de pacientes con AR, los niveles de IL10 se correlacionan con los títulos de factor reumatoideo (11) y la producción de dicho factor es dependiente de la IL-10 $(36,37)$. Esto sugiere que las altas concentraciones de IL-10 en AR, si bien son insuficientes para inactivar la cascada 
inflamatoria, pueden contribuir activamente a la progresión de la enfermedad promoviendo la autonmunidad.

Existe una gran variación entre individuos en la producción de IL-10. Un estudio reciente mostró que el componente genético en la variación de la producción de la IL-10 es aproximadamente del $70 \%$ (30). Las primeras investigaciones mostraron que la producción de IL-10 por parte de los leucocitos estimulados de sangre periférica es mayor en individuos con el alelo -1082G (14), y las personas homocigotas para el genotipo GCC/ GCC producen más IL-10, mientras que las de genotipos ACC/ACC, ACC/ATA y ATA/ATA la producen en baja cantidad $(17,38)$.

Diversos trabajos han analizado la relación de los polimorfismos de la IL-10 con la producción de IL-10 y su asociación con la presencia y gravedad de la AR, pero los resultados no han sido consistentes. En una población de 291 pacientes con AR en Holanda, Huizinga et al. no encontraron diferencias en la distribución de los genotipos en la posición -1082 al compararla con la del grupo control (39). Adicionalmente, en una cohorte de 138 mujeres con AR seguidas durante 12 años, las pacientes con el genotipo -1082AA presentaron radiográficamente menos daño articular a los 6 años que las que tenían el genotipo -1082GG (39). En un estudio realizado en Inglaterra, Coakley et al. no hallaron diferencias significativas en las frecuencias haplotícas o genotípicas entre controles y pacientes con AR y síndrome de Felty, una variedad de AR caracterizada por esplenomegalia y trombocitopenia (40). En una población de pacientes franceses con AR de inicio reciente, Cantagrel et al. no encontraron asociación con los alelos ni con los haplotipos en la posición -1082, así como tampoco con la gravedad de la enfermedad (41). De igual manera, en una población española, Martínez et al. no encontraron diferencias significativas en la distribución de la frecuencia de los haplotipos $-1082,-819$ y -592 entre pacientes y controles (42). Por el contario, Padyukov et al. informaron mayor prevalencia en mujeres suecas con AR comparadas con mujeres sanas del genotipo -1082AA, pero no así en hombres. No pudimos confirmar este hallazgo en nuestro estudio. Además, estos autores tampoco encontraron asociación con la presencia de factor reumatoideo (43). Hajeer et al. no encontraron asociación de estos polimorfismos de nucleótido simple con la AR, pero el haplotipo ACC, relacionado con baja producción de $\mathrm{IL}-10$, se asoció con la presencia de factor reumatoideo de la clase de $\lg A$ (44). En concordancia con los estudios mencionados, en nuestro trabajo no encontramos asociación de alelos, haplotipos o genotipos de los polimorfismos estudiados con la $\mathrm{AR}$, así como tampoco con el inicio de la enfermedad, la presencia de factor reumatoideo, los títulos altos o bajos del factor reumatoideo ni los antecedentes de reemplazo articular. A diferencia de tales hallazgos, Pawlik et al. encontraron una frecuencia significativamente mayor del genotipo GCC/GCC en 95 pacientes con esta enfermedad procedentes de Polonia, pero no encontraron asociación con la edad de inicio de la enfermedad ni con su actividad (45).

Otros investigadores han estudiado la asociación de otros polimorfismos del promotor de la IL-10 con la susceptibilidad a AR. El alelo IL-10.R2, asociado con mayor producción de IL-10, se presentó con una frecuencia significativamente mayor en pacientes con AR de Gran Bretaña y afroamericanos (46), hallazgo que no ha sido confirmado (47). Martínez et al., por el contrario, encontraron asociación entre artritis reumatoide y el alelo IL-10.G12 del microsatélite IL-10.G (42). Lard et al. no encontraron asociación de alelos ni genotipos del promotor -2849 A/G de la IL-10 con la incidencia de la $A R$, pero los pacientes con el genotipo -2849 AG/GG, asociado con alta producción de IL-10, tenían una mayor producción de factor reumatoideo y anticuerpos antipéptidos citrulinados de la clase de IgG (48). Otros polimorfismos de nucleótido simple en regiones más distales del promotor de la IL-10 (-2763, $3575,-6752,-6208)$ parecen también influir en la producción de IL-10 (49).

La herencia de los alelos de los genes de citocinas polimorfas está dramáticamente influenciada por la etnicidad. Existen grandes variaciones en la distribución de los polimorfismos del promotor de la IL-10 en diversas poblaciones, lo que puede explicar las diferentes asociaciones encontradas en los estudios. En el sur de la China, el alelo - 
1082A está presente en el $94 \%$ de la población (50) comparado con un 47 a $51 \%$ en la población del norte de Europa (51) y del $66 \%$ en nuestra población, lo cual explica la baja frecuencia del haplotipo GCC en las poblaciones orientales $(50,52)$. El genotipo GCC/GCC, relacionado con alta producción de IL-10, casi no aparece en poblaciones orientales y su presencia es poca en nuestra población $(6,7 \%)$ comparada con la europea (17). En general, la distribución de los alelos en la muestra estudiada tuvo una mayor similitud con la informada en España (42) que con la de países del norte de Europa $(45,46)$ o Asia $(50,52)$. Al igual que lo descrito en poblaciones caucásicas, el haplotipo GTA, presente en el 4\% de poblaciones asiáticas (50), no se encontró en nuestra población.

Hay pocos estudios sobre la distribución de polimorfismos de IL-10 en la población colombiana. Anaya JM et al. hallaron niveles significativamente más altos de IL-10 en pacientes con síndrome de Sjögren; adicionalmente, los mayores productores de IL-10 tenían más episodios de vasculitis cutánea y una mayor representación del alelo IL10.G9 (53). En el análisis de Henao et al. sobre los polimorfismos de citocinas en pacientes con diferentes tipos de tuberculosis, el genotipo 1082AA se asoció con tuberculosis pleural (54).

En este estudio, las frecuencias alélicas del polimorfismo -1082 en el grupo control se desviaron del equilibrio de Hardy-Weinberg por un exceso de heterocigotos en la población, hecho para el cual no tenemos una explicación clara , ya que la desviación no se presentó para los polimorfismos -819 y -592 que se encuentran en fuerte desequlibrio de unión. La influencia de factores desconocidos de tipo ambiental o genético en la segregación de los alelos, que pudieran enmascarar la detección del efecto del polimorfismo en esta población, podría considerarse como posible explicación. Estos factores pueden estar relacionados con presión selectiva hacia una respuesta inmune más efectiva frente a un ambiente con alta prevalencia de agentes infecciosos $(55,56)$, o a la existencia de desequilibrio de ligamiento con otras variantes polimórficas existentes en la región promotora del gen $(42,46)$.
Los estudios mencionados muestran que la IL10 tiene efectos inmunorreguladores y que los polimorfismos en la región promotora del gen de la IL-10 regulan su producción. Los resultados de esta investigación sugieren que en nuestra población los polimorfismos de nucleótido simple ubicados en las posiciones -1082 (G/A), $-819(\mathrm{C} / \mathrm{T})$ y $-592(C / A)$ no están asociados con la presencia y el curso de la artritis reumatoide; sin embargo, los resultados negativos obtenidos podrían relacionarse con el bajo poder del estudio debido al tamaño de la muestra, por lo que debería replicarse en otras poblaciones de distribución étnica similar para su confirmación.

\section{Agradecimientos}

Agradecemos a Carmen Cecilia Cabrales por su excelente asistencia técnica y manejo administrativo.

\section{Conflicto de intereses}

Ninguno.

\section{Financiación}

Este proyecto fue financiado por Colciencias (código 1102-04-12905) y la Universidad Industrial de Santander.

\section{Referencias}

1. Nepom GT. Papel del epítopo compartido DR4 en la selección y diferenciación de células $\mathrm{T}$ autorreactivas en la artritis reumatoide. Rheum Dis Clin North Am (Edición Española) 2001;3:41-53.

2. Newton JL, Harney SM, Wordsworth BP, Brown MA. A review of the MHC genetics of rheumatoid arthritis. Genes Immun 2004;5:151-7.

3. Deighton CM, Walter DJ, Griffiths ID, Robertos DF. The contribution of HLA to rheumatoid arthritis. Clin Genet 1989; 36:178-82.

4. Camargo JF, Correa PA, Vélez S, Anaya JM. Citocinas en artritis reumatoide. En: Ramírez LA, Anaya JM editores. Artritis Reumatoide. $2^{\mathrm{a}}$ edición. Bogotá: Editorial Edimeco S.A.; 2004. p.53-80.

5. Choy EH, Panayi GS. Cytokine pathways and joint inflammation in rheumatoid arthritis. $N$ Engl $J$ Med 2001;344:907-16.

6. Szekannez Z, Koch AE. Cytokines. In: Ruddy S, Harris ED, Sledge CB, Budd RC, Sergent JS. Textbook of

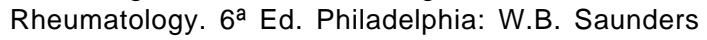
Company; 2001. p.275-87. 
7. Arend WP, Gabay C. Cytokines in the rheumatic diseases. Rheum Dis Clin North Am 2004;30:41-67.

8. Moore KW, de Waal Malefyt R, Coffman RL, O'Garra A. Interleukin-10 and the interleukin -10 Receptor. Annu Rev Immunol 2001;19:683-765.

9. Van Roon JA, Lafeber FP, Bijlsma JW. Synergistic activity of interleukin-4 and interleukin-10 in suppression of inflammation and joint destruction in rheumatoid arthritis. Arthritis Rheum 2001;44:3-12.

10. Katsikis PD, Chu CO, Brennan FM, Maini RN, Feldman M. Immunoregulatory role of interleukin 10 in rheumatoid arthritis. J Exp Med 1994;179:1517-27.

11. Cush JJ, Splawski JB, Thomas R, McFarlin JE, Schulza-Koops H, Davis LS et al. Elevated interleukin 10 levels in patients with rheumatoid arthritis. Arthritis Rheum 1995;38:96-104.

12. Rousset G, Garcia E, Defrance T, Peronne C, Vezzio N, Hsu DH et al. Interleukin-10 is a potent growth and differentiation factor for activated human B lymphocytes. Proc Natl Acad Sc USA 1992;89:1890-3.

13. Westendorp RGJ, Langermans JA, Huizinga TWJ, Elouali AH, Verweij CL, Boomsma DI et al. Genetic influence on cytokine production and fatal meningococcal disease. Lancet 1997;349:170-3.

14. Turner DM, Williams DM, Sankarán D, Lazarus M, Sinnott PJ, Hutchinson IV. An investigation of polymorphism in the interleukin-10 gene promoter. Eur J Inmunogenet 1997;24:1-8.

15. Asadullah K, Sterry W, Volk HD. Interleukin-10 therapy. Review of a new approach. Pharmacol Rev 2003;55:241-69.

16. Westendorp RG, van Dunne G FM, Kirkwood TB, Helmerhorst FM, Huizinga TW. Optimizing human fertility and survival (letter). Nat Med 2002;7:873.

17. Suárez A, Castro $\mathbf{P}$, Alonso R, Mozo L, Gutiérrez C. Interindividual variations in constitutive interleukin-10 messenger RNA and protein levels and their association with genetic polymorphisms. Transplantation 2003;75:711-7.

18. Panayi GS, Corrigall VM, Pitzalis C. Patogenesis de la artritis reumatoide. El papel de las células $\mathrm{T}$ y otras bestias. Rheum Dis Clin North Am (Edición Española) 2001;3:55-76

19. Van Roon JA, Wijngaarden S, Labefer FP, Damen C, Van der Winkel JG, Bulsma JW. Interleukin 10 treatment of patients with rheumatoid arthritis enhances Fc $\gamma$ receptor expression on monocytes and responsiveness to immune complex stimulation. J Rheumatol 2003;30:648-51.

20. Arnett FC, Edworthy SM, Bloch DA, McShane DJ, Fries JF, Cooper NS et al. The American Rheumatism Association 1987 revised criteria for the classification of rheumatoid arthritis. Arthritis Rheum 1988;31:315-24.
21. Miller SA, Dykes DD, Polesky HF. A simple salting out procedure for extracting DNA from human nucleated cells. Nucl Acids Res 1988;16:1215.

22. Mullighan CG, Marshall SE, Bunce M, Welsh KI. Variation in immunoregulatory genes determines the clinical phenotype of common variable immunodeficiency. Genes Immun 1999;1:137-48.

23. Daniel WW. Bioestadística. Base para el análisis de las ciencias de la salud. 4⿳亠丷a edición. México D.F: Edit. Limusa Wiley; 2002. p.571-657.

24. Schneider S, Roessli D, Excoffier L. Arlequin version 2000. A software for population genetics data analysis. Genetics and Biometry Laboratory. Geneva: University of Geneva; 2000.

25. Hartl DL, Clark AG. Principles of population genetics. 2nd ed. Sinauer Sunderland-Massachsetts: Associate Inc Publishers; 1989. p.293-8.

26. Houssiau FA, Lefebre $C$, van den Berghe $M$, Lambert M, Devogelaer JP, Reanuld JC. Serum interleukin 10 titers in systemic lupus erythematosus reflect disease activity. Lupus 1995;4:393-5.

27. Llorente L, Richaud-Patin Y, Fior R, Alcocer-Varela $\mathrm{J}$, Wijdenes $\mathrm{J}$, Fourrier BM et al. In vivo production of interleukin- 10 by non-T cells in rheumatoid arthritis, Sjogren's Syndrome and systemic lupus erythematosus: A potential mechanism of B lymphocyte hyperactivity and autoimmunity. Arthritis Rheum 1994;37:1647-55.

28. Anaya JM, Correa PA, Herrera M, Eskdale J, Gallagher G. IL-10 influences autoimmune response in primary Sjögren's syndrome and is linked to IL-10 gene polymorphism. J Rheumatol 2002;29:1874-6.

29. Fortis C, Foppoli M, Gianotti L, Galle L, Citterio G, Consogno $\mathrm{G}$ et al. Increased Interleukin-10 serum levels in patients with solid tumors. Cancer Lett 1996;104:1-5.

30. Westendorp RD, Langermans JA, Huizinga TW, Verweij CL, Sturk A. Genetic influence on cytokine production in meningococcal disease. Lancet 1997;349:1912-3.

31. Koss K, Satsangi J, Fanning GC, Welsh KI, Jewell DP. Cytokine (TNF $\alpha$, LT $\alpha$ and IL-10) polymorphisms in inflammatory bowel diseases and normal controls: differential effects on production and allele frequencies. Genes Immun 2000;1:185-90.

32. Reich K, Garbe C, Blaschke V, Maurer C, Middel P, Westphal G et al. Response of psoriasis to interleukin10 associated with suppression of cutaneous type 1 inflammation, downregulation of the epidermal interleukin 8/CXCR2 pathway and normalization of keratinocyte maturation. J Invest Dermatol 2001;116:319-29.

33. Lim S, Crawley E, Woo P, Barnes PJ. Haplotype associated with low interleukin 10 production in patients with severe asthma. Lancet 1998;352:113. 
34. Schulze-Koops H, Davis LS, Kavanaugh AF, Lipsky PE. Elevated cytokine messenger RNA levels in the peripheral blood of patients with rheumatoid arthritis suggest different degrees of myeloid cell activation. Arthritis Rheum 1997;40:639-47.

35. Isomäki $\mathbf{P}$, Luukkainen $\mathbf{R}$, Saario $\mathbf{R}$, Toivanen $\mathbf{P}$, Punnonen J. Interleukin-10 functions as an antiinflammatory cytokine in rheumatoid synovium. Arthritis Rheum 1996;39:386-95.

36. Perez L, Orte J, Brieva JA. Terminal differentiation of spontaneous rheumatoid factor-secreting $B$ cells from rheumatoid arthritis patients depends on endogenous interleukin-10. Arthritis Rheum 1995;38:1771-6.

37. Reparon-Schuijt CC, van Esch WE, van Kooten C, Levarht EW, Breedveld FC, Verweij CL. Functional analysis of rheumatoid factor producing $B$ cells from the synovial fluid of rheumatoid arthritis patients. Arthritis Rheum 1998;41:2211-20.

38. Hoffmann SC, Stanley ER, Cox ED, Craighead N, DiMercurio BS, Koziol DE et al. Association of cytokine polymorphic inheritance and in vitro cytokine production in anti CD3/CD28- stimulated peripheral blood lymphocytes. Transplantation 2001;72:1444-50.

39. Huizinga TW, Keijsers V, Yanni G, Hall M, Ramage $\mathbf{W}$, Lanchbury $\mathbf{J}$ et al. Are differences in interleukin 10 production associated with joint damage? Rheumatology (Oxford) 2000;39:1180-8.

40. Coakley G, Mok CC, Hajeer AH, Ollier WER, Turner D, Sinnott PJ. Interleukin-10 promoter polymorphisms in rheumatoid arthritis and Felty's syndrome. $\mathrm{Br} \mathrm{J}$ Rheumatol 1998;37:988-91.

41. Cantagrel A, Navaux F, Loubet-lescoulié $\mathbf{P}$, Nourhasehemi F, Enault G, Abbal M et al. Interleukin1â, interleukin-1 receptor antagonist, interleukin-4 and interleukin-10 gene polymorphisms. Arthritis Rheum 1999;42:1093-100.

42. Martínez A, Pascual M, Pascual-Salcedo D, Balsa A, Martin J, de la Concha EG. Genetic polymorphisms in Spanish rheumatoid arthritis patients: an association and linkage study. Genes Immun 2003;4:117-21.

43. Padyukov L, Hytönen AM, Smolnikova M, HahnZoric M, Nilsson N, Hanson LA et al. Polymorphism in promoter region of $\mathrm{IL}-10$ gene is associated with rheumatoid arthritis in women. J Rheumatol 2004;31: 422-5.

44. Hajeer AH, Lazarus M, Turner D, Mageed RA, Vencovsky J, Sinnott $\mathbf{P}$ et al. IL-10 gene promoter polymorphisms in rheumatoid arthritis. Scan $J$ Rheumatol 1998;27:142-5.

45. Pawlik A, Kurzawski M, Szlarz BG, Hercynska M, Drozdzik M. Interleukin-10 promoter polymorphism in patients with rheumatoid arthritis. Clin Rheumatol 2005;24:480-4

46. Eskdale J, McNicholl J, Wordsworth P, Jonas B, Huizinga T, Field $\mathbf{M}$ et al. Interleukin-10 microsatellite polymorphisms and IL-10 locus alleles in rheumatoid arthritis susceptibility (letter). Lancet 1998;352:1282-3.

47. MacKay K, Milicic A, Lee D, Tikly M, Laval S, Shatford $\mathbf{J}$ et al. Rheumatoid arthritis susceptibility and interleukin-10. Rheumatology 2003;42:149-53.

48. Lard LR, van Gaalen FA, Schonkeren JJ, Pieterman EI, Toeken G, Kos K et al. Association of the -2849 interleukin-10 promoter polymorphism with autoantibody production and joint destruction in rheumatoid arthritis. Arthritis Rheum 2003;48:1841-8.

49. Mörmann M, Rieth H, Hua TD, Assohou C, Roupelieva M, Hu SL et al. Mosaics of gene variations in the Interleukin-10 gene promoter affect interleukin10 production depending on the stimulation used. Genes Immun 2004;5:246-55.

50. Mok CC, Lanchbury JS, Chan DW, Lau CS. Interleukin-10 promoter polymorphisms in southern Chinese patients with systemic lupus erythematosus. Arthritis Rheum 1998;41:1090-5.

51. Reynard MP, Turner D, Navarrete CV. Allele frequencies of polymorphisms of the tumor necrosis factor- $\alpha$, interleukin-10, interferon- $\gamma$ and interleukin -2 genes in a North European Caucasoid group from the UK. Eur J Immunogenet 2000;27:241-9.

52. Origuchi, Kawasaki E, Ide A, Kamachi M, Tanaka F, Ida $\mathrm{H}$ et al. Correlation between interleukin 10 promoter region polymorphisms and clinical manifestations in Japanese patients with Sjogren's syndrome. Ann Rheum Dis 2003;62:1117-8.

53. Anaya JM, Correa PA, Herrera M, Eskdale, Gallagher G. Interleukin 10 (IL-10) influences autoinmmune response in primary Sjögrens's syndrome and is linked to IL-10 gene polymorphisms. J Rheumatol 2002; 29:1874-6

54. Henao MI, Montes C, París SC, García LF. Cytokines gene polymorphisms in Colombian patients with different clinical presentations of tuberculosis. Tuberculosis 2006;86:11-9

55. Larcombe L, Rempel JD, Dembinski I, Tinckam K, Rigatto C, Nickerson P. Differential cytokine genotype frequencies among Canadian aboriginal and Caucasian populations. Genes Immun 2005;6:140-4.

56. Torkildsen O, Utsi E, Harbo HF, Mellgren SI, Vedeler CA, Myhr KM. Ethnic variations of IL-10 polymorphisms in a Sami and Norwegian population. Scan J Immunol 2005;62:71-4. 